\title{
Capsaicin upregulates HDAC2 via TRPV1 and impairs neuronal maturation in mice
}

\author{
Sung Eun Wang ${ }^{1,5}$, Seung Yeon Ko ${ }^{1,5}$, Yong-Seok Kim ${ }^{1,2,5}$, Sungsin $\mathrm{Jo}^{3}$, Seung Hoon Lee ${ }^{1}$, Sung Jun Jung ${ }^{1,4}$ \\ and Hyeon Son ${ }^{1,2}$
}

Transient receptor potential vanilloid 1 (TRPV1) affects mood and neuroplasticity in the brain, where its role is poorly understood. In the present study we investigated whether capsaicin (8-methyl-N-vanillyl-trans-6-nonenamide), an agonist of TRPV1, induced chromatin remodeling and thereby altered gene expression related to synaptic plasticity. We found that capsaicin treatment resulted in upregulation of histone deacetylase 2 (HDAC2) in the mouse hippocampus and HDAC2 was enriched at Psd95, synaptophysin, GLUR1, GLUR2 promoters. Viral-mediated hippocampal knockdown of HDAC2 induced expression of Synapsin I and prevented the detrimental effects of capsaicin on Synapsin I expression in mice, supporting the role of HDAC2 in regulation of capsaicin-induced Synapsin I expression. Taken together, our findings implicate HDAC2 in capsaicin-induced transcriptional regulation of synaptic molecules and support the view that HDAC2 is a molecular link between TRPV1 activity and synaptic plasticity.

Experimental \& Molecular Medicine (2018) 50, e455; doi:10.1038/emm.2017.289; published online 9 March 2018

\section{INTRODUCTION}

Capsaicin (8-methyl-N-vanillyl-trans-6-nonenamide), a component of red chili peppers, is an agonist of transient receptor potential vanilloid type1 (TRPV1) whose activation increases pain by exciting primary sensory neurons in the peripheral nervous system. ${ }^{1}$ TRPV1 activation by agonists induces L-type calcium channel-dependent apoptosis of primary rat cortical neurons ${ }^{2}$ and impairs proliferation of neural progenitor cells in the hippocampus of young mice. ${ }^{3}$ These findings suggest that capsaicin is harmful to neural development in the brain. However, the effects of capsaicin on neuronal maturation and synaptic plasticity have not been adequately explored.

Preclinical studies show that TRPV1 mediates anxiety- and depression-related behaviors. TRPV1 activation by systemic injection of agonists such as capsaicin and resiniferatoxin elicits anxiogenic responses and depression-related behaviors, whereas its antagonists induce anxiolytic- and antidepressantlike effects in rodents. ${ }^{4-7}$ Besides, direct injection of capsaicin or capsazepine in the hippocampus induces anxiety- or anxiolytic-like effects. ${ }^{8,9}$ These effects are accompanied by impaired hippocampal neurogenesis through epigenetic regulation. ${ }^{10,11} \mathrm{~A}$ number of previous studies have demonstrated that chromatin-remodeling factors including histone deacetylases (HDACs) play a key role in adult neurogenesis and synaptic plasticity and hence in cognition and mood. ${ }^{12,13}$ HDACs repress gene transcription by removing acetyl groups from histone tails. ${ }^{12}$ We recently demonstrated that the antidepressant-like effects induced by TRPV1 deficiency are associated with HDAC2 downregulation. ${ }^{7}$ However, TRPV1 activation produces depression-like effects with an increase in HDAC2 expression. However, there is no evidence that TRPV1 activation by agonists induces HDAC2 upregulation and thereby alters synaptic molecules or neuronal maturation in the hippocampus.

We now report that systemic capsaicin administration for 2 weeks in adult mice led to a reduction in number of mature neurons as well as of neural proliferation in the dentate gyrus (DG) of the hippocampus. It also elevated the amount and activity of HDAC2 in the hippocampus, which in turn decreased synaptic plasticity-related molecules, as HDAC2 repressed the activity of their promoters. To investigate the effect of HDAC2 on synaptic density, we infected primary

\footnotetext{
${ }^{1}$ Graduate School of Biomedical Science and Engineering, Hanyang Biomedical Research Institute, College of Medicine, Hanyang University, Seongdong-gu, Seoul, Republic of Korea; ${ }^{2}$ Department of Biochemistry and Molecular Biology, College of Medicine, Hanyang University, Seongdong-gu, Seoul, Republic of Korea; ${ }^{3}$ Hanyang University Hospital for Rheumatic Disease, Hanyang University, Seongdong-gu, Seoul, Republic of Korea and ${ }^{4}$ Department of Physiology, College of Medicine, Hanyang University, Seongdong-gu, Seoul, Republic of Korea

${ }^{5}$ These authors contributed equally to this work.

Correspondence: Professor H Son, Department of Biochemistry and Molecular Biology, College of Medicine, Hanyang University, 222 Wangsimni-ro, Seongdong-gu, Seoul 04763, Republic of Korea.

E-mail: hyeonson@hanyang.ac.kr

Received 13 June 2017; revised 29 August 2017; accepted 27 September 2017
} 
hippocampal neurons with lenti-shHDAC2 to reduce HDAC2 expression. We found that knockdown of HDAC2 reversed the decrease in synaptic density induced by TRPV1 activation. Taken together, these results suggest that TRPV1 activation impairs synaptic plasticity by upregulating HDAC2.

\section{MATERIALS AND METHODS}

Mice

All experiments were conducted with 8-10-week-old male C57BL/6J (Charles River Korea, Seoul, Korea) mice. Trpv1 ${ }^{-/-}$mice $^{14}$ were obtained from the Jackson Laboratory (Bar Harbor, ME, USA). They were backcrossed into the C57BL/6J (Charles River Korea) background, and mice of the N7 or higher generation were used. Heterozygous breeders were crossed to generate wild type, heterozygous and knockout littermates, and genotypes were determined using PCR analysis. Mice were housed four per cage in a temperatureand humidity-controlled environment (lights on 0700-1900) with access to food and water ad libitum. All animal experiments were approved by the Institutional Animal Care and Use Committee of the Hanyang University and were performed in accordance with relevant guidelines and regulations.

\section{Culture of primary hippocampal neurons}

Hippocampi from day 14 C57BL/6 mouse embryos were rapidly and aseptically removed from brains into ice-cold $\mathrm{Ca}^{2+} / \mathrm{Mg}^{2+}$-free Hanks' balanced salt solution (HBSS), followed by removal of meninges and mincing into small pieces. The hippocampal tissue was then digested in $0.25 \%$ EDTA-trypsin (Gibco, Grand Island, NY, USA) and dissolved in neurobasal medium (Gibco) containing 10\% (vol/vol) fetal bovine serum, $75 \mathrm{mmol}^{-1}$ L-glutamine and $0.1 \%$ penicillinstreptomycin, and centrifuged at $200 \mathrm{~g}$ for $1 \mathrm{~min}$. The pelleted cells were gently resuspended in culture medium and plated at 40000 50000 cells per $\mathrm{cm}^{2}$ on poly-L-lysine-coated $(25 \mathrm{mg} / \mathrm{ml}$ in phosphatebuffered saline (PBS); Sigma, St Louis, MO, USA) and laminin-coated
(10 $\mathrm{mg} \mathrm{ml}^{-1}$ in PBS; Sigma) culture dishes. Hippocampal cultures were grown for 1 day in neurobasal medium containing $10 \%$ (vol/vol) fetal boine serum, $75 \mathrm{mmol}^{-1} \mathrm{~L}$-glutamine and $0.1 \%$ penicillinstreptomycin. The medium was changed the following day to neurobasal medium supplemented with $0.02 \%$ B27 serum-free supplement, $75 \mathrm{mmol}^{-1} \mathrm{~L}$-glutamine and $0.1 \%$ penicillin-streptomycin antibiotic mixture. Cultures were maintained for 7-12 days at $37^{\circ} \mathrm{C}$ in a $5 \% \mathrm{CO}_{2} / 95 \%$ air-humidified incubator. Neurons were used after 7-12 days.

\section{Drugs}

Capsaicin (8-methyl-N-vanillyl-trans-6-nonenamide, M2028, Sigma) was administered intraperitoneally to mice at $0.001,0.01$ and $0.1 \mathrm{mg} \mathrm{kg}^{-1} \mathrm{~d}^{-1}$ for 2 weeks. Vehicle (PBS with $10 \%$ ethanol and $10 \%$ Tween 20) was used as a control. All administration processes were conducted during the dark cycle. Embryo hippocampal primary neurons were treated with capsaicin for $48 \mathrm{~h}$. For in vitro drug treatment, capsaicin was prepared at $1 \mathrm{mg} \mathrm{ml}^{-1}$ stock solution in vehicle and diluted in fresh medium at a final concentration of $1 \mu \mathrm{m}$. The same volume of vehicle was added into the medium of nontreated cultures as a control.

\section{HDAC enzyme activity}

The HDAC-Glo 2 Assay and HDAC-Glo class IIa Assay (Promega, Madison, WI, USA) were used to determine lysate activities. Luminescence was measured using a Panomics Luminometer (Gentaur, Kampenhout, Belgium).

\section{BrdU administration and cell counting}

Mice were injected with bromodeoxyuridine (BrdU; $50 \mu^{-1} \mathrm{~g}^{-1}$; Sigma) at $0.75 \mathrm{mg} \mathrm{ml}^{-1}$. BrdU was injected on the first 3 days of the experiment to examine the survival of neurons. The number of $\mathrm{BrdU}(+)$ cells was counted in the subgranular zone (SGZ) or granular cell layers using a fluorescence microscope (Leica Microsystems,

Table 1 Primary antibodies used in WB, ICC, IHC and ChIP.

\begin{tabular}{|c|c|c|c|c|}
\hline Antigen & Manufacturer & Species, type & Catalog number & Dilution \\
\hline BrdU & Abcam/Cambridge, UK & Rat monoclonal & ab6326 & $1: 300$ \\
\hline HDAC2 & Abcam/Cambridge, UK & Rabbit polyclonal & ab16032 & $1: 1000$ \\
\hline HDAC4 & Abcam/Cambridge, UK & Rabbit polyclonal & ab12172 & $1: 1000$ \\
\hline HDAC5 & Abcam/Cambridge, UK & Rabbit polyclonal & ab1439 & $1: 1000$ \\
\hline pSer10acK14 Histone H3 & Upstate/NY, USA & Rabbit polyclonal & \#07-081 & $1: 1000$ \\
\hline Acetyl-Histone H3 & Millipore/CA, USA & Rabbit polyclonal & \#06-599 & $1: 1000$ \\
\hline Histone H3 & Upstate/NY, USA & Mouse monoclonal & \#05-499 & $1: 1000$ \\
\hline GluR1 & Upstate/NY, USA & Rabbit polyclonal & \#06-306 & $1: 1000$ \\
\hline GluR2 & Millipore/CA, USA & Rabbit polyclonal & AB1768-I & $1: 1000$ \\
\hline NR1 & Upstate/CA, USA & Rabbit monoclonal & \#06-311 & $1: 1000$ \\
\hline GFP & Santa Cruz/TX, USA & Rat monoclonal & sc-101536 & $1: 200$ \\
\hline
\end{tabular}

Abbreviations: BrdU, bromodeoxyuridine; ChIP, chromatin immunoprecipitation; HDAC, histone deacetylase; ICC, immunocytochemistry; IHC, immunohistochemistry; NeuN, neuronal nuclei; WB, western blotting. 
Table 2 Primer sequences for qPCR.

\begin{tabular}{|c|c|c|}
\hline Gene & 5'-Forward-3' & 5'-Reverse-3' \\
\hline Actb (Actin) (ChIP) & GCCGCTGTGGCGTCCTATAA & TGAGGTACTAGCCACGAGAG \\
\hline Synapsin2 (ChIP) & GGCTTTCCTTCCСTCCACAC & TGTTAGCGAGGGAGCAGTGG \\
\hline Psd95 (ChIP) & ССССТАССССТССТGAGAAT & GAGGGGAAGGAGAAGGTTGG \\
\hline GLURI (ChIP) & GGAGGAGAGCAGAGGGAGAG & TTCCTGCAATTCCTTGCTTG \\
\hline Creb (ChIP) & CTACACCAGCTTCCCCGGT & ACGGAAACAGCCGAGCTC \\
\hline Hdac2 (mRNA) & AAATCAGCTCAGAAAGGCCA & CCAAGGACAATAGTGGTGAG \\
\hline Synapsin 1 (mRNA) & CACCAGGATGAAGACAAGCA & GTCGTTGTTGAGCAGGAG \\
\hline Actb (mRNA) & CGGAACCGCTCATTGCC & АСССАСАСТGTGCCСАTСTА \\
\hline
\end{tabular}

Abbreviation: qPCR, quantitative real-time PCR.

Wetzlar, Germany) at $\times 400$, plus video camera and LEICA IM50 software (Leica Microsystems) in six-to-eight coronal $25 \mu \mathrm{m}$ sections spaced $400 \mu \mathrm{m}$ apart per mouse. BrdU(+) cells in SGZ and granular cell layer sections were counted by an experimenter blind to the study code. Data are expressed as the average number of immunoreactive cells, and reported as means \pm s.e.m. For statistical analysis, unpaired $t$-tests were used.

\section{Extraction of histones}

Histones were acid-extracted as described previously. ${ }^{15}$ Hippocampi were homogenized in lysis buffer (\#9803, Cell Signaling, Danvers, MA, USA) and the pellets were resuspended in $0.4 \mathrm{~N} \mathrm{H}_{2} \mathrm{SO}_{4}$ and incubated on ice for $20 \mathrm{~min}$. Samples were washed with acidified acetone and centrifuged at 14000 r.p.m. The histone-containing pellets were dissolved in $10 \mathrm{~mm}$ Tris $(\mathrm{pH}$ 8.0) and subjected to western blot analysis.

\section{Immunohistochemistry and western blot analysis}

Mice were perfused with $4 \%$ paraformaldehyde in PBS for $20 \mathrm{~min}$ and processed for histology. Brains were rapidly removed and immediately frozen and stored at $-70^{\circ} \mathrm{C}$. Serial sections $(25 \mu \mathrm{m}$ per section) were cut coronally through the entire anteroposterior extent of the hippocampus. To detect BrdU and neuronal nuclei (NeuN), sections were processed as described. ${ }^{16}$ For other types of immunofluorescence labeling, sections were incubated in $10 \%$ normal goat serum and $0.3 \%$ Triton X-100 for $1 \mathrm{~h}$ at room temperature. They were then incubated overnight at $4{ }^{\circ} \mathrm{C}$ in $0.1 \mathrm{M}$ PBS, pH 7.4, containing $0.3 \%$ Triton $\mathrm{X}-100$ (PBST), and washed in PBST. Primary antibodies used for immunohistochemistry are described in Table 1 . To detect primary antibodies, cells were incubated in PBS containing Cy3-labeled (Jackson Immunoresearch, West Grove, PA, USA), Cy5-labeled (Jackson Immunoresearch) or fluorescein isothiocyanate (FITC)-labeled (Invitrogen, Carlsbad, CA, USA) secondary antibodies for $2 \mathrm{~h}$ at room temperature $\left(25^{\circ} \mathrm{C}\right)$ and photographed with a confocal microscope (Leica Microsystems). Z-stack images were acquired by optical sectioning, and LAS AF software (Leica Microsystems) was used to sharpen images, adjust brightness and contrast levels, and compose Z-projection (maximum intensity) and channel-merged figures. Cells were counterstained with 4',6-diamidino-2-phenylindole in addition to the four immunological markers. For western blot analysis, samples were prepared as previously described. ${ }^{16}$ The primary antibodies used for western blot analysis are described in Table 1.

\section{Chromatin immunoprecipitation assays}

Chromatin immunoprecipitation (ChIP) assays were performed as previously described. ${ }^{17}$ The antibodies used are presented in Table 1. Immunoprecipitated DNA samples were resuspended in distilled $\mathrm{H}_{2} \mathrm{O}$ and used for real-time PCR (CFX96 Touch Real-Time PCR Detection System, Bio-Rad Laboratories, Foster City, CA, USA). Primer sequences are given in Table 2, and were previously reported. ${ }^{17}$ Input and immunoprecipitated DNAs were PCR-amplified in triplicate in the presence of SYBR Green (Bio-Rad Laboratories). The fraction of genomic DNA co-precipitated with specific antibody was calculated as: $\Delta C_{\mathrm{T}}=C_{\mathrm{T}}$ (genomic input) $-C_{\mathrm{T}}$ (specific antibody), where $C_{\mathrm{T}}$ (genomic input) and $C_{\mathrm{T}}$ (specific antibody) are the mean threshold cycles of PCR performed in duplicate on DNA samples from the genomic input samples and the antibody-treated samples, respectively. $2^{\Delta \mathrm{CT}}$ target values were calculated for each antibody. ${ }^{18}$ Enrichment was assessed by quantitative real-time PCR (qPCR) for the seven promoters and normalized to the values obtained with the Actin promoter.

\section{RT-PCR and qPCR}

RNA was extracted from hippocampi with Trizol reagent (Sigma). Reverse transcription was performed with Improm-II (Promega), $1 \mu \mathrm{g}$ of total RNA and oligonucleotide-dT primer. qPCR was performed on a CFX96 Touch Real-Time PCR Detection System (Bio-Rad Laboratories). The primers used to amplify cDNAs are given in Table 2. Ct values for each sample were obtained using CFX Manager Software version 3.0 (Bio-Rad Laboratories). The expression of each gene was normalized to the amount of $\beta$-actin. Normalized expression values were averaged, and average fold changes were calculated.

\section{Construction of a shRNA (shRNA-expressing vector) and lentivirus production}

For gene silencing, short hairpin RNAs (shRNAs) were cloned into the pLB lentiviral vector (Addgene, Cambridge, MA, USA), as described. ${ }^{19}$ Their sequences were as follows: Luciferase shRNA (shLuc, control shRNA), 5'-CTTCGAAATGTCCGTTCGGTT-3'; HDAC2 shRNA (shHDAC2) 5'-CCCAATGAGTTGCCATATAAT-3'. Lentivirus was produced by co-transfection of shLuc, shHDAC2 with the compatible packaging plasmids, pMD2.G and psPAX2 into 293T cells (ATCC, Manassas, VA, USA) by the calcium-phosphate method, as described by the RNAi Consortium (http://www.broadinstitute.org). 
a

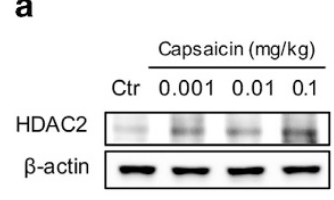

b

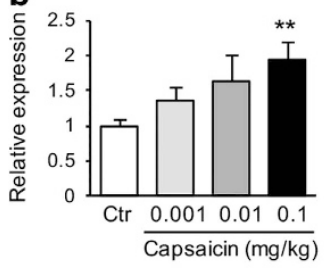

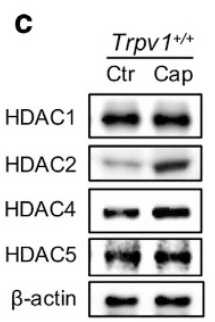

d

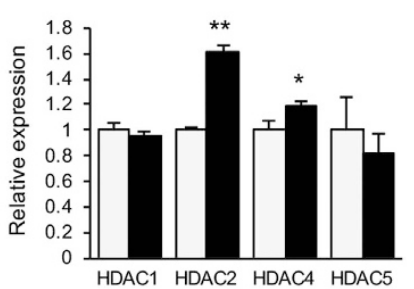

e

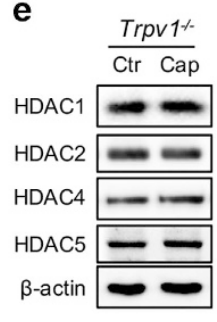

Control Capsaicin

f

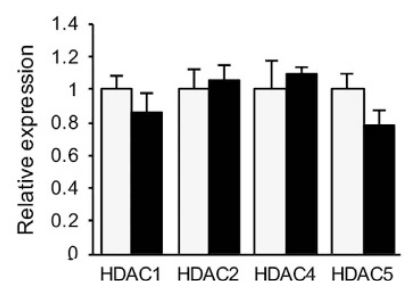

Figure 1 Capsaicin elevates expression of HDAC2 in a TRPV1-dependent manner in mouse hippocampus. (a) Representative blots of HDAC2 levels in the hippocampus of WT mice treated with capsaicin for 2 weeks in a dose-dependent manner. (b) Quantification of the experiments in a showing significant elevation of HDAC2 in hippocampal lysates of capsaicin-treated mice at the dose of $0.1 \mathrm{mg} \mathrm{kg}^{-1}$ ( $n=3$ animals per group). (c) Representative blots of HDAC levels in the hippocampus of WT mice treated with capsaicin for 2 weeks. (d) Quantification of the experiments in c showing significant elevation of HDAC2 and HDAC4 by TRPV1 activation ( $n=3$ animals per group). (e) Representative blots of HDAC in the hippocampus of Trpv1 $1^{-1}$ mice treated with capsaicin for 2 weeks. (f) Quantification of the results in e. There was no difference in the HDAC level between control Trpv1 ${ }^{-/}$mice and capsaicin-treated Trpv1 $1^{-/}$mice $(n=3$ animals per group). Values are means \pm s.e.m. Unpaired $t$-test; ${ }^{*} P<0.05,{ }^{* *} P<0.01$. HDAC, histone deacetylase; TRPV1, transient receptor potential vanilloid 1 ; WT, wild type.
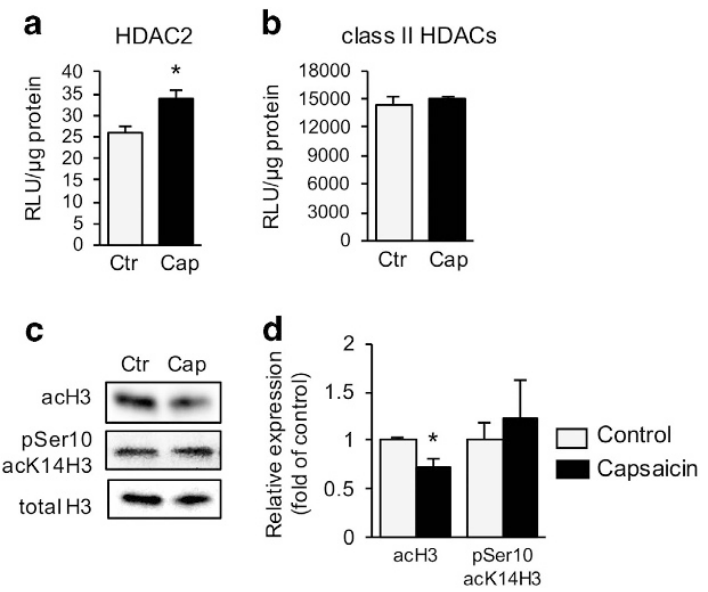

Figure 2 Capsaicin increases HDAC2 activity in the mouse hippocampus. (a) Increased relative luciferase units (RLUs) of HDAC2 activity in hippocampal lysates of capsaicin-treated mice. Luciferase activities were normalized to protein content $(n=3$ animals per group). (b) The RLU of class II HDAC activity was not changed by capsaicin ( $n=3$ animals per group). (c) Representative blots showing histone acetylation in hippocampal histone extracts. (d) Quantification of c showing decreased histone H3 acetylation in capsaicin-treated mice. Loading was normalized to total H3 $(n=3$ animals per group). Values are means \pm s.e.m. Unpaired $t$-test; ${ }^{*} P<0.05$. HDAC, histone deacetylase.

\section{Statistical analyses}

Data distributions were checked for normality and equality of variance. All experiments were carried out at least three times, and data consistency was observed in repeated experiments. Wherever possible, the experimenter conducting the data analysis was blind to the treatment conditions of the animals. Differences between groups were analyzed by two-way analysis of variance followed by the Bonferroni adjustment for multiple comparisons between groups when assessing the effect of drugs on the virus. GraphPad Prism5.0 (GraphPad Software, La Jolla, CA, USA) was used for statistical analysis. $P<0.05$ was considered statistically significant. Biochemical data are presented as means \pm s.e.m. of multiple independent experiments performed in triplicate.

\section{RESULTS}

Capsaicin induces HDAC2 in the mouse hippocampus

We investigated whether capsaicin administration changed the amounts of HDAC1, 2, 4 and 5 in the mouse hippocampus in which these HDACs are abundant. ${ }^{20}$ To investigate the threshold dose of capsaicin for inducing epigenetic effects in the hippocampus, we examined the dose-dependent effects of capsaicin administration. We found that HDAC2 expression had a tendency to increase in a dose-dependent manner with a significant increase at the dose of $0.1 \mathrm{mg} \mathrm{kg}^{-1}$ (Figure 1a and b). Therefore, we used a $0.1 \mathrm{mg} \mathrm{kg}^{-1}$ dose of capsaicin for 2 weeks, which also induced impairment of adult hippocampal neurogenesis as shown in a previous study. ${ }^{3}$ HDAC2 protein increased upon capsaicin treatment compared with vehicletreated controls (Figure 1c and d), whereas HDAC4 protein increased only slightly (Figure $1 \mathrm{c}$ and $\mathrm{d}$ ). To ask whether the increases in HDAC2 and 4 were dependent on capsaicininduced TRPV1 activation, we administered capsaicin to Trp $v 1^{-/-}$mice. Levels of HDAC2 and 4 in the hippocampus of $\operatorname{Tr} p v 1^{-1-}$ mice were unaffected by capsaicin administration (Figure 1e and f), indicating that the increased levels of HDAC2 
and 4 were the result of TRPV1 activation. We then tested the activities of HDAC2 and class II HDACs including HDAC4. HDAC2 activity increased, but the activity of class II HDACs was not altered (Figure 2a and b). Because HDAC2 activity was elevated, its target substrate, histone $\mathrm{H} 3$, was less acetylated (Figure $2 \mathrm{c}$ and d).However, the level of histone H3 Ser10 and acetylation at Lys14 (pSer10-acK14H3), known to be associated with hippocampal learning and memory processes, ${ }^{21}$ was not altered by capsaicin (Figure $2 \mathrm{c}$ and $\mathrm{d}$ ). These results demonstrated that elevation of HDAC2 regulated histone modification in the hippocampus through TRPV1-dependent signaling.

Capsaicin downregulates levels of postsynaptic molecules through HDAC2 in the hippocampus

A previous report has suggested that HDAC2 represses synaptic plasticity-related genes by binding to their promoters. ${ }^{17}$ Even though HDAC2 lacks a DNA-binding domain, its transcriptional repressor complex including CoREST and $\mathrm{mSin} 3 \mathrm{~A}$ has a role in gene silencing as suggested previously. ${ }^{22}$ Having seen that capsaicin increased HDAC2 in the hippocampus, we explored its effect on HDAC2 binding to the promoters of synaptic molecules. We examined the association of HDAC2 with the promoter regions of synapsin2 (Syn2), synaptophysin (SYP), GLUR1, GLUR2 and Psd95, and included the QUOTE -actin promoter as control. Capsaicin increased HDAC2 binding to the SYP, GLUR1 and GLUR2 promoters (Figure 3a). We also noted that HDAC2 had a strong tendency to bind to the Psd95 promoter in the capsaicin-treated
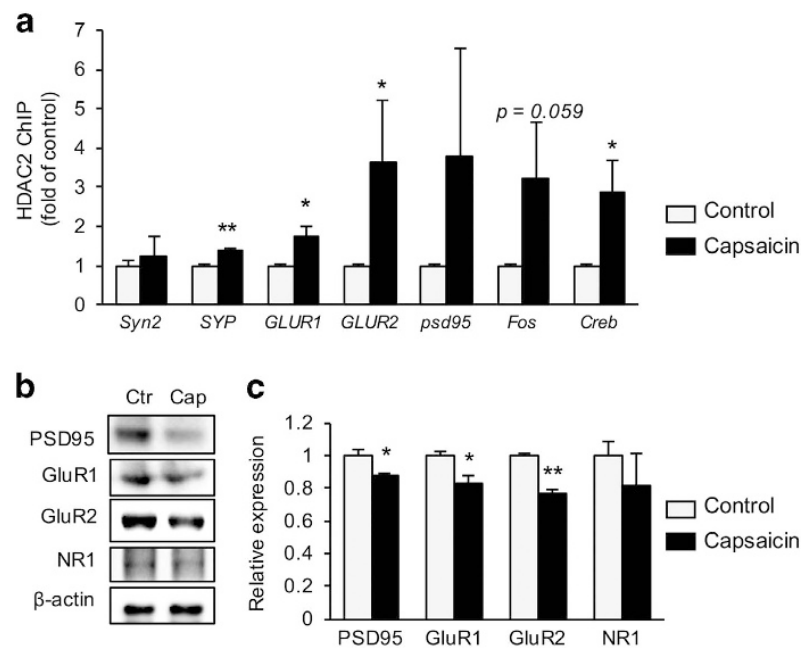

Figure 3 Capsaicin downregulates the expression of synaptic molecules through HDAC2 in the hippocampus. (a) ChIP assays. TRPV1 activation increases binding of HDAC2 to synaptic plasticityrelated gene promoters in the hippocampus $(n=3$ animals per group). (b) Representative blots of synaptic molecules in the hippocampi of WT and capsaicin-treated mice. (c) Quantification of the results in b. PSD95, GluR1 and GluR2 protein levels are reduced in the hippocampus of capsaicin-treated mice $(n=3$ animals per genotype). Values are means \pm s.e.m. Unpaired $t$-test; ${ }^{*} P<0.05,{ }^{* *} P<0.01$. ChIP, chromatin immunoprecipitation; HDAC, histone deacetylase; TRPV1, transient receptor potential vanilloid 1; WT, wild type. hippocampus (Figure 3a). Consistent with the repression of HDAC2 by capsaicin in the mouse hippocampus, we observed reduced levels of PSD95, GluR1 and GluR2 (Figure 3b and c). We further found that TRPV1 activation affected the binding of HDAC2 to the promoters of Fos and Creb (Figure 3a), which play an important role in neuronal differentiation and neurogenesis. ${ }^{23}$ Taken together, our findings indicated that increasing HDAC2 by capsaicin treatment led to a reduction of synaptic molecules.

\section{Capsaicin reduces synaptic density in primary hippocampal neurons}

To assess the effect of an increase in HDAC2 triggered by capsaicin on synaptic plasticity, we infected primary hippocampal neurons with lenti-shHDAC2 (Figure 4a) and confirmed that HDAC2 expression was attenuated at the mRNA level (Figure 4b). In addition, capsaicin alone increased Hdac2 mRNA levels in neurons infected with the control virus (Figure 4b). This upregulation of Hdac2 transcripts was accompanied by reduced Synapsin I transcription (Figure 4c). Capsaicin reduced the number of synaptic puncta, and this reduction was prevented by knockdown of HDAC2 (Figure $4 \mathrm{~d}$ and e). These results indicated that capsaicin disrupted synaptic function by increasing HDAC2.

Capsaicin impairs the maturation as well as the proliferation of neurons in the DG

To further demonstrate the effect of capsaicin-induced TRPV1 activation on neurogenesis including neuronal proliferation and differentiation, we administrated BrdU to mice for three consecutive days before administering capsaicin for 2 weeks (Figure 5a) and examined the number of BrdU-positive cells in the SGZ and DG (Figure 5b). Capsaicin reduced the proliferation in the SGZ (Figure 5c) and survival of newborn neurons in the DG of adult mice $(-23 \%$; Figure $5 \mathrm{~d})$. We then examined expression of nuclear antigen (NeuN), a marker of neuronal maturation. The number of NeuN-positive cells co-labeled with $\mathrm{BrdU}$ was reduced by capsaicin $(-36 \%$, Figure $5 \mathrm{e} ;-31 \%$, Figure 5f). These results suggest that capsaicin-induced TRPV1 activation impaired neuron maturation in the DG of adult mice.

\section{DISCUSSION}

Here we have shown that TRPV1 activation induced by capsaicin impaired neuron maturation and synaptic plasticity in the hippocampus. We suggest that capsaicin-induced TRPV1 activation upregulates HDAC2 and decreases acetylation of histone $\mathrm{H} 3$, resulting in reduced expression of synaptic molecules. Furthermore, TRPV1 activation leads to loss of synapses, which is reversed by knocking down HDAC2 in primary hippocampal neurons.

Previous work has revealed that animals treated with TRPV1 agonists and antagonists exhibit depression- and antidepression-like behaviors, respectively. ${ }^{4-6,24,25}$ However, the molecular mechanisms that underlie these emotional responses have not been elucidated. There are a number of 
a
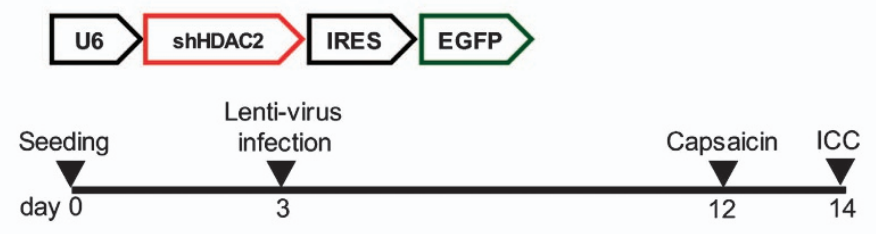

b

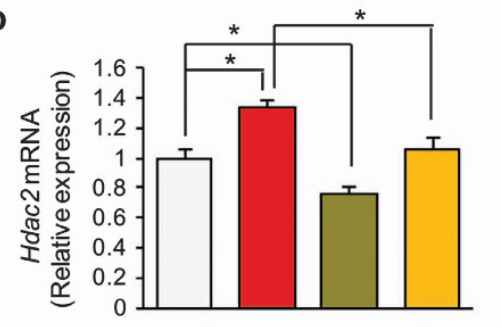

d

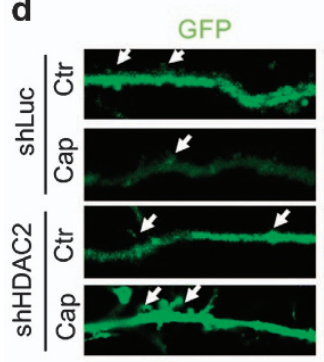

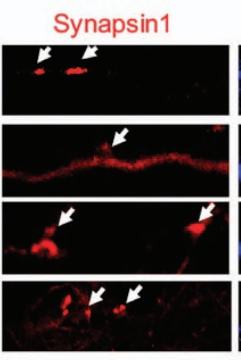

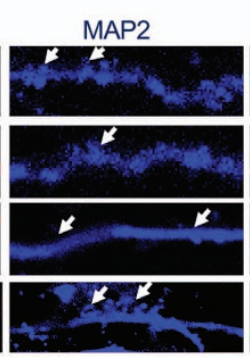

shLuc-Ctr

shLuc-Cap

shHDAC2-Ctr

shHDAC2-Cap c

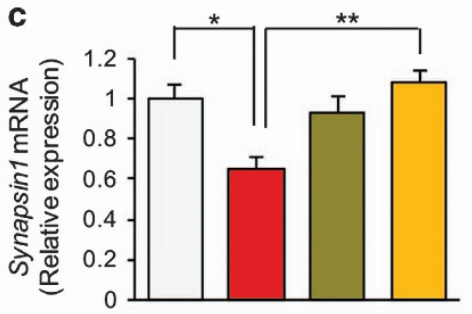

e

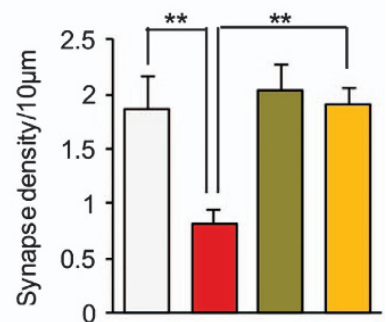

Figure 4 Capsaicin reduces synapse density in mouse hippocampal primary neurons. (a) Targeting strategy for deleting HDAC2 mRNA. The mouse U6 promoter was used to drive the expression of HDAC2-shRNA (upper). Experimental design (lower). (b) Mouse Hdac2 and $\beta$-actin mRNA levels were quantified by qPCR ( $n=3-4$ per group). Unpaired two-tailed $t$-test; ${ }^{*} P<0.05$ (c) Mouse Synapsin I and $\beta$-actin mRNA levels were quantified by qPCR ( $n=3$ per group). Two-way analysis of variance (ANOVA; main effect of interaction $F_{1,8}=14.19$, $P=0.0055$; virus $F_{1,8}=7.892, \quad P=0.0229$ ) followed by Bonferroni's multiple comparison test. ${ }^{*} P<0.05,{ }^{* *} P<0.01$. (d) Tripleimmunostaining of lenti-shLuc- or lenti-shHDAC2-infected neurons with anti-synapsin1, anti-MAP2 and anti-GFP antibodies. (e) Quantification of the number of synapsin1(+) MAP2(+) synaptic puncta per $10 \mu \mathrm{m}$ of dendrite in lenti-shRNA-infected neurons $\left(n=14-20\right.$ ). Two-way ANOVA (main effect of interaction $F_{1,60}=4.758, P=0.0331$; virus $F_{1,60}=9.34, P=0.0033$; drug $F_{1,60}=8.195$, $P=0.0058$ ) followed by Bonferroni's multiple comparison test. ${ }^{* *} P<0.01$. Scale bar $5 \mu \mathrm{m}$. Values are means \pm s.e.m. GFP, green fluorescent protein; HDAC, histone deacetylase; qPCR, quantitative polymerase chain reaction; shRNA, short hairpin RNA.

reports of the functional roles of HDAC2 in the maturation of neural cells, ${ }^{26,27}$ especially in neurogenesis ${ }^{28}$ and synaptic plasticity. ${ }^{17}$ According to our recent work, ${ }^{7}$ TRPV1 loss of function induces downregulation of HDAC2 in the DG, leading to elevated expression of neurogenesis- and synaptic plasticity-related genes, which contributes to stress-resilient behavior. However, increase of HDAC2 expression in the DG induces depression-like behaviors, which were blocked by HDAC2 knockdown. ${ }^{7}$ In line with this, we confirmed above that TRPV1 activity stimulated HDAC2 upregulation and impaired neuronal maturation. We also found that TRPV1 activity reduced the survival of neural progenitor cells as well as neural proliferation in the DG. Taken together, these results suggest that TRPV1 regulates neurogenesis and synaptic plasticity through HDAC2-mediated chromatic remodeling.

TRPV1 is mainly expressed in the sensory neurons of the peripheral nervous system as well as the hypothalamus, amygdala and hippocampus in the central nervous system. ${ }^{29}$ Systemic injection of capsaicin may produce pain-related effects through TPRV1 expressed in sensory neurons. These sensory inputs from the periphery terminate in the spinal cord, where integration and hypersensitivity can be established. Spinal outputs connect with the limbic system including the hippocampus. ${ }^{30}$ However, the capsaicin dosage $\left(0.1 \mathrm{mg} \mathrm{kg}^{-1}\right.$, intraperitoneally) used in our experiment is low relative to that used in pain induction or behavioral response change. ${ }^{31}$ Therefore, the indirect action of capsaicin in our study may not be significant.

In humans, TRPV1 may not be exposed to the dose of capsaicin that was used in this study. However, TRPV1 could be activated by endogenous ligands such as endocannabinoids or several lipoxygenase metabolites as well as capsaicin intake. $^{32,33}$ Especially, endocannabinoids are produced by stress-induced glucocorticoids in the brain. ${ }^{34}$ Therefore, TRPV1 activation could be achieved by lipid metabolites and stress-induced endocannabinoids at even lower concentrations of capsaicin that used in this study.

In summary, activation of TRPV1 and expression of HDAC2 in response to exogenous stimuli may constitute the molecular 
a $\mathrm{BrdU}$

\begin{tabular}{lllllc}
$\mathbf{\nabla}$ & & & \\
& & Capsaicin i.p. & Sacrifice \\
\hline 1 & 2 & 3 & 4 & 2 weeks & 18
\end{tabular}
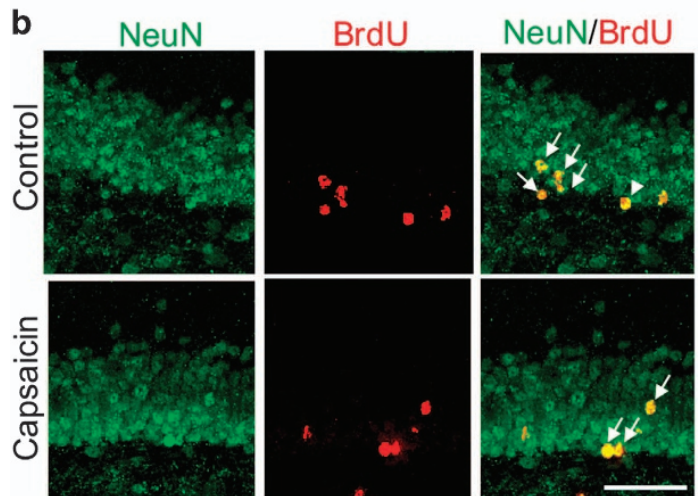

c

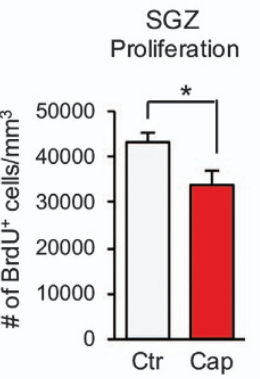

d $\quad$ DG
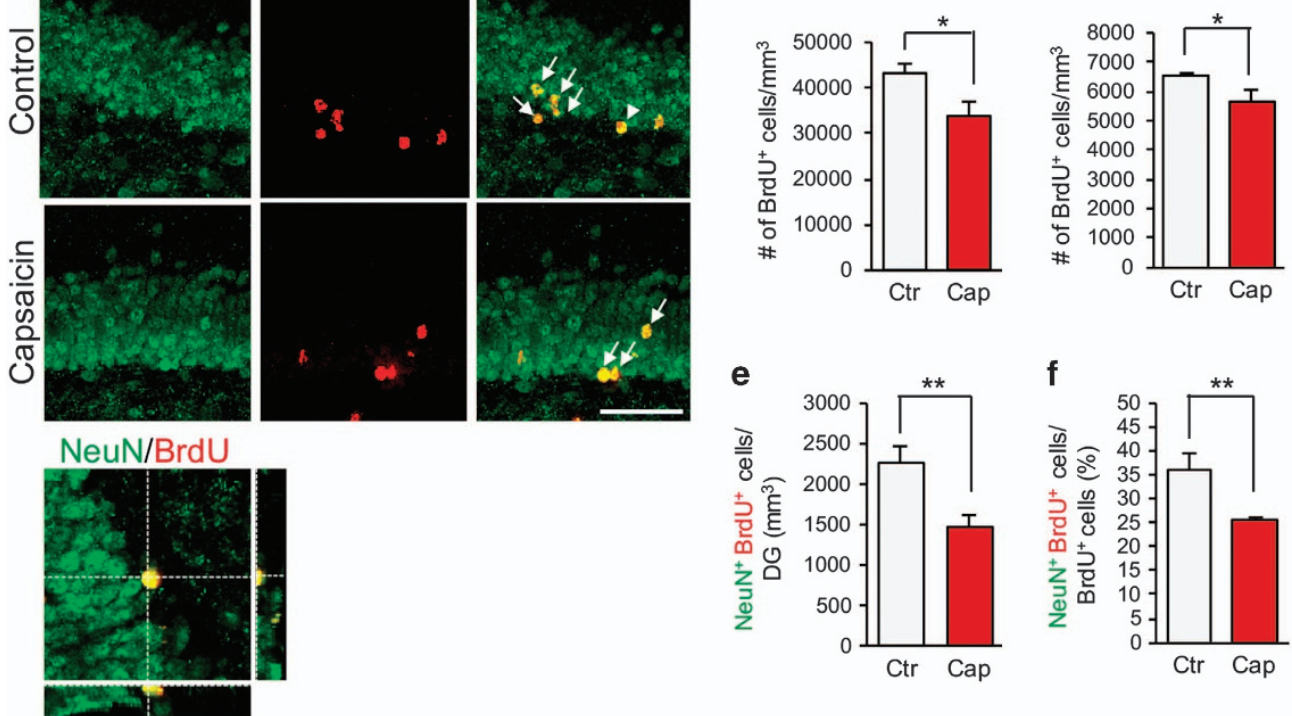

Figure 5 Capsaicin impairs the survival of neural progenitor cells as well as proliferation in the hippocampus. (a) Experimental design of the cell survival studies following injection with capsaicin for 14 days starting on day 4 . BrdU ( $\left.50 \mathrm{mg} \mathrm{kg}^{-1}\right)$ was given three times every $24 \mathrm{~h}$ during an initial period of 3 days. (b) Labeling of NeuN (green) in the DG in the survival paradigm (upper). High-power confocal image of a cell (arrowhead in b) labeled with BrdU (red) and expressing NeuN (green). The bottom and right panels are images merged across the $x$ - and $z$-axes, respectively (lower). (c-f) Quantifications of the BrdU- and NeuN-labeled cells in the SGZ or DG ( $n=4$ animals per group). Scale bar $50 \mu \mathrm{m}$. Values are means \pm s.e.m. Unpaired $t$-test; ${ }^{*} P<0.05,{ }^{* *} P<0.01$. DG, dentate gyrus; NeuN, neuronal nuclei; SGZ, subgranular zone.

mechanisms that together contribute to neuroplasticity. Our findings provide evidence that TRPV1 modulates HDAC2 binding to the promoters of synaptic molecules and suggest that epigenetic mechanisms play an important role in TRPV1mediated neurodegeneration.

\section{CONFLICT OF INTEREST}

The authors declare no conflict of interest.

\section{ACKNOWLEDGEMENTS}

This work was supported by the research fund of Hanyang University (HY-2017), a grant from the Medical Research Center (NRF-

2017R1A5A2015395), and Basic Science Research Program though the National Research Foundation of Korea (NRF) funded by the Ministry of Education (No. 2016R1A2B2006474, 2017R1A6A3A01005765).

\section{PUBLISHER'S NOTE}

Springer Nature remains neutral with regard to jurisdictional claims in published maps and institutional affiliations.

1 Moalem G, Grafe P, Tracey DJ. Chemical mediators enhance the excitability of unmyelinated sensory axons in normal and injured peripheral nerve of the rat. Neuroscience 2005; 134: 1399-1411.
2 Shirakawa H, Yamaoka T, Sanpei K, Sasaoka H, Nakagawa T, Kaneko S. TRPV1 stimulation triggers apoptotic cell death of rat cortical neurons. Biochem Biophys Res Commun 2008; 377: 1211-1215.

3 Kong KH, Kim HK, Song KS, Woo YS, Choi WS, Park HR et al. Capsaicin impairs proliferation of neural progenitor cells and hippocampal neurogenesis in young mice. J Toxicol Environ Health A 2010; 73: 1490-1501.

4 Abdelhamid RE, Kovacs KJ, Nunez MG, Larson AA. Depressive behavior in the forced swim test can be induced by TRPV1 receptor activity and is dependent on NMDA receptors. Pharmacol Res 2014; 79: 21-27.

5 Navarria A, Tamburella A, lannotti FA, Micale V, Camillieri G, Gozzo L et al. The dual blocker of FAAH/TRPV1 N-arachidonoylserotonin reverses the behavioral despair induced by stress in rats and modulates the HPA-axis. Pharmacol Res 2014; 87: 151-159.

6 Kasckow JW, Mulchahey JJ, Geracioti TD Jr. Effects of the vanilloid agonist olvanil and antagonist capsazepine on rat behaviors. Prog Neuropsychopharmacol Biol Psychiatry 2004; 28: 291-295.

7 Wang SE, Ko SY, Jo S, Choi M, Lee SH, Jo H et al. TRPV1 regulates stress responses through HDAC2. Cell Rep 2017; 19: 401-412.

8 Hakimizadeh E, Oryan S, Hajizadeh Moghaddam A, Shamsizadeh A, Roohbakhsh A. Endocannabinoid system and TRPV1 Receptors in the dorsal hippocampus of the rats modulate anxiety-like behaviors. Iran $\mathrm{J}$ Basic Med Sci 2012; 15: 795-802.

9 Santos CJ, Stern CA, Bertoglio LJ. Attenuation of anxiety-related behaviour after the antagonism of transient receptor potential vanilloid type 1 channels in the rat ventral hippocampus. Behav Pharmacol 2008; 19: 357-360.

10 Hsieh J, Eisch AJ. Epigenetics, hippocampal neurogenesis, and neuropsychiatric disorders: unraveling the genome to understand the mind. Neurobiol Dis 2010; 39: 73-84.

11 Mill J, Petronis A. Molecular studies of major depressive disorder: the epigenetic perspective. Mol Psychiatry 2007; 12: 799-814. 
12 Krishnan V, Nestler EJ. Linking molecules to mood: new insight into the biology of depression. Am J Psychiatry 2010; 167: 1305-1320.

13 Graff J, Mansuy IM. Epigenetic codes in cognition and behaviour. Behav Brain Res 2008; 192: 70-87.

14 Caterina MJ, Leffler A, Malmberg AB, Martin WJ, Trafton J, Petersen-Zeitz KR et al. Impaired nociception and pain sensation in mice lacking the capsaicin receptor. Science 2000; 288: 306-313.

15 Yu IT, Park JY, Kim SH, Lee JS, Kim YS, Son H. Valproic acid promotes neuronal differentiation by induction of proneural factors in association with H4 acetylation. Neuropharmacology 2009; 56: 473-480.

16 Lee JS, Jang DJ, Lee N, Ko HG, Kim H, Kim YS et al. Induction of neuronal vascular endothelial growth factor expression by CAMP in the dentate gyrus of the hippocampus is required for antidepressant-like behaviors. I Neurosci 2009; 29: 8493-8505.

17 Guan JS, Haggarty SJ, Giacometti E, Dannenberg JH, Joseph N, Gao J et al. HDAC2 negatively regulates memory formation and synaptic plasticity. Nature 2009; 459: 55-60.

18 Bararia D, Trivedi AK, Zada AA, Greif PA, Mulaw MA, Christopeit M et al. Proteomic identification of the MYST domain histone acetyltransferase TIP60 (HTATIP) as a co-activator of the myeloid transcription factor $\mathrm{Cl}$ EBPalpha. Leukemia 2008; 22: 800-807.

19 Jo S, Lee H, Kim S, Hwang EM, Park JY, Kang SS et al. Inhibition of PCGF2 enhances granulocytic differentiation of acute promyelocytic leukemia cell line HL-60 via induction of HOXA7. Biochem Biophys Res Commun 2011; 416: 86-91.

20 Kazantsev AG, Thompson LM. Therapeutic application of histone deacetylase inhibitors for central nervous system disorders. Nat Rev Drug Discov 2008; 7: 854-868.

21 Reul JM, Hesketh SA, Collins A, Mecinas MG. Epigenetic mechanisms in the dentate gyrus act as a molecular switch in hippocampus-associated memory formation. Epigenetics 2009; 4: 434-439.

22 Delcuve GP, Khan DH, Davie JR. Roles of histone deacetylases in epigenetic regulation: emerging paradigms from studies with inhibitors. Clin Epigenet 2012; 4: 5.

23 West AE, Greenberg ME. Neuronal activity-regulated gene transcription in synapse development and cognitive function. Cold Spring Harb Perspect Biol 2011; 3: a005744.

24 You IJ, Jung YH, Kim MJ, Kwon SH, Hong SI, Lee SY et al. Alterations in the emotional and memory behavioral phenotypes of transient receptor potential vanilloid type 1-deficient mice are mediated by changes in expression of 5-HT(1)A, GABA(A), and NMDA receptors. Neuropharmacology 2012; 62: 1034-1043.

25 Socala K, Wlaz P. Evaluation of the antidepressant- and anxiolytic-like activity of alpha-spinasterol, a plant derivative with TRPV1 antagonistic effects, in mice. Behav Brain Res 2016; 303: 19-25.
26 MacDonald JL, Roskams AJ. Histone deacetylases 1 and 2 are expressed at distinct stages of neuro-glial development. Dev Dyn 2008; 237: 2256-2267.

27 Montgomery RL, Hsieh J, Barbosa AC, Richardson JA, Olson EN. Histone deacetylases 1 and 2 control the progression of neural precursors to neurons during brain development. Proc Natl Acad Sci USA 2009; 106: $7876-7881$.

28 Jawerka M, Colak D, Dimou L, Spiller C, Lagger S, Montgomery RL et al. The specific role of histone deacetylase 2 in adult neurogenesis. Neuron Glia Biol 2010; 6: 93-107.

29 Menigoz A, Boudes M. The expression pattern of TRPV1 in brain. $J$ Neurosci 2011; 31: 13025-13027.

30 O'Neill J, Brock C, Olesen AE, Andresen T, Nilsson M, Dickenson AH. Unravelling the mystery of capsaicin: a tool to understand and treat pain. Pharmacol Rev 2012; 64: 939-971.

31 Guler AD, Rainwater A, Parker JG, Jones GL, Argilli E, Arenkiel BR et al. Transient activation of specific neurons in mice by selective expression of the capsaicin receptor. Nat Commun 2012; 3: 746.

32 Takayama Y, Uta D, Furue H, Tominaga M. Pain-enhancing mechanism through interaction between TRPV1 and anoctamin 1 in sensory neurons. Proc Natl Acad Sci USA 2015; 112: 5213-5218.

33 Szolcsanyi J, Sandor Z. Multisteric TRPV1 nocisensor: a target for analgesics. Trends Pharmacol Sci 2012; 33: 646-655.

34 Popoli M, Yan Z, McEwen BS, Sanacora G. The stressed synapse: the impact of stress and glucocorticoids on glutamate transmission. Nat Rev Neurosci 2011; 13: 22-37.

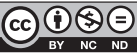

This work is licensed under a Creative Commons Attribution-NonCommercial-NoDerivs $\quad \mathbf{4 . 0}$

International License. The images or other third party material in this article are included in the article's Creative Commons license, unless indicated otherwise in the credit line; if the material is not included under the Creative Commons license, users will need to obtain permission from the license holder to reproduce the material. To view a copy of this license, visit http://creativecommons.org/licenses/by-nc-nd/4.0/

(C) The Author(s) 2018 